\title{
Patient Safety in Spine Surgery: Regarding the Wrong-Site Surgery
}

\author{
Seung-Hwan Lee ${ }^{1}$, Ji-Sup Kim ${ }^{1}$ Yoo-Chul Jeong ${ }^{1}$, Dae-Kyung Kwak ${ }^{1}$, Ja-Hae Chun², Hwan-Mo Lee ${ }^{1}$ \\ ${ }^{1}$ Department of Orthopedic Surgery, Yonsei University College of Medicine, Seoul, Korea \\ ${ }^{2}$ Department of Quality Improvement and Patient Safety, Severance Hospital, Yonsei University College of Medicine, Seoul, Korea
}

Patient safety regarding wrong site surgery has been one of the priority issues in surgical fields including that of spine care. Since the wrong-side surgery in the DM foot patient was reported on a public mass media in 1996, the wrong-site surgery issue has attracted wide public interest as regarding patient safety. Despite the many wrong-site surgery prevention campaigns in spine care such as the operate through your initial program by the Canadian Orthopaedic Association, the sign your site program by the American Academy of Orthopedic Surgeon, the sign, mark and X-ray program by the North American Spine Society, and the Universal Protocol program by the Joint Commission, the incidence of wrong-site surgery has not decreased. To prevent wrong-site surgery in spine surgeries, the spine surgeons must put patient safety first, complying with the hospital policies regarding patient safety. In the operating rooms, the surgeons need to do their best to level the hierarchy, enabling all to speak up if any patient safety concerns are noted. Changing the operating room culture is the essential part of the patient safety concerning spine surgery.

Keywords: Patient safety; Wrong-site surgery; Spine

\section{Introduction}

In general, the events of wrong-site surgery occur rarely [1-3]. When it does occur, however, it is considered a sentinel event which may cause severe damages to patients and physicians physically as well as mentally. In spine surgery field, as with other fields of specialty, it is understood that the right surgery is done to correct the patient. However, wrong-site surgery is still an ongoing problem in spine surgery, just as in other surgery fields $[1,4]$. The incidence of neurological complication or infection after spine surgery cannot be zero, even with a lot of efforts made to reduce them [5]. The incidents of wrong-site surgery may be a preventable if the medical staff follows the right method to avoid such events.

\section{History}

When the incidents of wrong-site surgery occurred in the past, they were often resolved in the name-blame-shame tradition on the surgeon or the surgical team, without any cause analysis and monetary rewards. These methods are not helpful today in resolving problems [6]. "To err is human," the report published by the Institute of Medicine (IOM) in 1999 [7], has drawn attention of not only physicians but also the general public. This report does not disclose the limitations in the spine field, but the issues of medical errors, infections, and technical problems of surgery can be considered in regards to the spine surgery. As the wrong-site surgery is a preventable medical error, by using a quality improvement process, many medical

Received Sep 22, 2012; Revised Oct 4, 2012; Accepted Oct 4, 2012

Corresponding author: Hwan-Mo Lee

Department of Orthopedic Surgery, Yonsei University College of Medicine,

50 Yonsei-ro, Seodaemun-gu, Seoul 120-749, Korea

Tel: +82-2-2228-2191, Fax: +82-2-363-1139, E-mail: hwanlee@yuhs.ac 
organizations have carried in-depth studies regarding the issue.

In spite of the controversies in the IOM report, orthopedic surgeons have been interested in the wrong-site surgery issues for a long time. The Canadian Orthopaedic Association (COA) [8] and the American Academy of Orthopedic Surgeons (AAOS) [9] have sought preventive measures through case analysis, by forming the task force on wrong-site surgery. It was calculated that the cumulative probability of having a wrong-site surgery at least once in an orthopedic surgeon's life-time career was 25\% [10].

\section{Sign Your Site Program and Sign, Mark, And X-ray Program}

The AAOS has proposed the Sign Your Site program in prevention of wrong-site surgery. The AAOS started the Sign your site (SYS) campaign in 1997 using the checklist including the initials signed by the surgeons on the surgical sites. In 2000, $78 \%$ of the American orthopedic surgeons were aware of the SYS program, with only $46 \%$ actually using the program [10]. The first SYS program was modified for the verification of the appropriate side and level of spine pathology [11]. A check list is used as a system memory and for documentation of instrumentation.

The North American Spine Society (NASS) modified the SYS program of AAOS into a more detailed comprehensive one that contained appropriate level confirmation and side of the spine pathology for surgical intervention. NASS developed a sign, mark, and X-ray (SMaX) program for identification of exact patient and operation level. The SMaX program contains three key steps for patient safety in spine care $[12,13]$.

1. Process of patient identification and confirmation of medical records including imaging studies and informed consents.

2. Surgical site markings.

3. Verification of the spine pathology level during operation.

This process involves: 1) completion of pre-op checklist, 2) surgical site marking done by the surgeon on the neck or trunk prior to the skin incision, 3) taking the intra-operative X-ray by using metal markers in order to verify the spine level. In this method of verifying the pathology level intra-operatively, X-ray must be taken by using metal markers such as towel chip or K-wires. Therefore, SMaX checklist (Fig. 1), recommended by the NASS, allows physicians to systematically review the information regarding patients, so that the spine surgeries can be done safely and successfully.

It is desirable that the informed consents are obtained by surgeons or the teaching staff, and the informed consents must have names, medical record numbers, diagnoses, name of surgeries, site and side of proposed surgery, and lastly, the signatures of responsible surgeon and patient. This informed consent must be shared with surgeon as well as anesthesiologist, assistant, and scrub nurse. The surgical marking must be done by the surgeon by putting his/her initial on the surgical site prior to the surgery and also by having the patient participate, and when the surgery is unilateral, sign of left or right must also be indicated (Fig. 2).

The last step of the SMaX program is accurately verifying the spine pathology level by using a portable radiography intra-operatively. The lumbosacral junction sites can be verified only through finger palpation, but verifying the site by using portable radiography with metal marker is much more accurate [14]. Also in cervical spine, approximately $17 \%$ can approach the wrong level if anatomical landmark such as carotid tubercle is being used [15]. As such, portable radiography must be used to verify the areas of lesion. In thoracic spine, it is safe to verify the level by counting the number of ribs on radiography, however, it requires attention when there is cervical rib or size of the 12th rib is small [16].

In the lumbar spine, attention is required in verifying the level if there is a transitional vertebra. In cervical spine and lumbar spine, lateral radiography is useful for level verification. In thoracic spine, AP radiography is frequently used for level verification, since the spinous process lies in distal direction and ribs can cover the view. When verifying the level by using AP radiography in thoracic spine, mistakes may be reduced by obtaining an image including the 12th rib.

\section{The Joint Commission and the Joint Commission International}

The Joint Commission (JC) has discovered 278 cases of wrong-site surgeries in the sentinel events reported from 1995 to 2003 . Of these, $58 \%$ of the cases were wrong-side 


\section{Sign, Mark \& X-ray (SMaX)}

A Checklist for Safety

- Involve the patient in confirming the operative site either through informed consent or during the actual marking. Surgeons are encouraged to personally obtain informed consent. Copies of the operative permit/informed consent form should state the site and side of surgery and be shared with the patient, surgeon, anesthesiologist, assistant or scrub nurse and circulating nurse.

o Sign your name to the operative site.

- Each member of the operative team should verify the correct site.

o Verify that X-rays and medical records are for the correct patient, as well as confirming the identity of the patient.

- Each of the following items should be double-checked against the marked site:

- Medical record

○ X-rays and other imaging studies (marked " $L$ " or " $R$ " to prevent being placed backwards on the light box)

- Informed consent

- Operating room/anesthesia record

- Consider having your assistant or scrub nurse always stand opposite the side where the surgeon should stand.

- Consider or suggest an intraoperative X-ray during surgery, after exposure using markers that do not move to confirm the vertebral level to be operated. Consider a radiology reading.

\section{Complete all the items on this page. Relying on a single preventive effort only can result in errors!}

Patient Name:

Physician:

Procedure(s):

Date:

Signature of Person Completing the Checklist:

$\square$ 2001, North American Spine Society

Fig. 1. Sign, mark, and X-ray preoperative check list (NASS) (Reprinted from: Sign, mark \& X-ray (SMaX): a checklist for safety, with permission from North American Spine Society) [12]. 
surgeries, $12 \%$ were wrong patients, and $10 \%$ were wrong procedures $[13,17]$. The analysis revealed that the $12 \%$ of all wrong-site surgeries occurred in the institutions where 2 out of 3 items were carried out, and these 3 items were 1) patient verification, 2) surgical site marking, 3) time-out. On the other hand, wrong-site surgeries did not occur in the institutions where all 3 items were carried out. Based on this result, JC has implemented Universal Protocol for patient surgical site identification starting in July of 2004 [18]. The Universal Protocol is composed of 1) patient identification, 2) surgical site marking, 3) time-out. The process of patient identification and surgical site marking are similar to the SMaX program from NASS, and the time-out process is performed by all operating team members just prior to the beginning of operation by verifying the patient's name, medical record number, name of surgery, surgical site, and equipments/devices to be used.

SYS and SMaX programs were incorporated into JC methodology, and both AAOS and NASS have endorsed the Universal Protocol [13]. Universal Protocol is included in the International Patient Safety Goal (IPSG) of Joint Commission International (JCI) and all JCI-accredited hospitals must follow this protocol. IPSG 1 states that the standards on correct patient identification and IPSG 4 are concerned with the correct-site, correct-procedure, and correct- patient surgery. The time-out process needs to be conducted at the location of the procedure, just prior to the beginning of the procedures, and involve the entire operative team [19]. According to the IPSG guidelines, the organization determines how the time-out process is to be documented, and usually a checklist is needed for

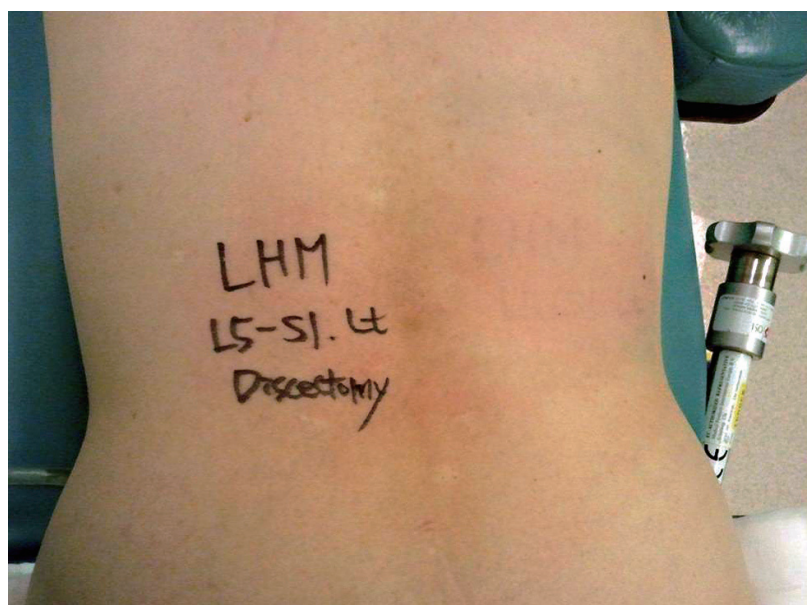

Fig. 2. Example of preoperative surgical site marking: left side discectomy is planned at L5-S1 level. confirmation of the process (Fig. 3).

\section{Sign In-Time Out-Sign Out Guidelines: WHO}

In 2008, World Health Organization (WHO) proposed guidelines for the safety of surgical patients [20]. Haynes and others designed and used the checklist with 19 items for sign in-time out-sign-out [21]. The sign in process is carried out before induction, and in addition to the patient identification, there is a verification process of any potential anesthetic problems, allergy, and pulse oxymetry. The time out process is carried out just prior to the incision by the entire team participating in the surgery, and in addition to the items stated in the universal protocol of JC, the expected amount of blood loss and administration of prophylactic antibiotics are also checked. The sign out process is carried out before the patient leaves the operating room, and it includes the verification of the right surgery as planned, needles and sponge counts, and surgical specimen.

Since the use of the WHO guideline checklists in the hospitals around the world, the mortality rate has declined to $0.8 \%$ from $1.5 \%$. The patient complications have also decreased to $7.0 \%$ from $11.0 \%$ [21].

\section{Has the Rate of Wrong-Site Surgery Decreased Since the Introduction of the Campaigns?}

The Canadian Orthopaedic Association and the Canadian Medical Protective Association monitored if the operate through your initials program was effective in reducing the rate of wrong-site surgery in the orthopaedic field. They compared the data for seven years before the initiation of the program with the data for seven years after the program and concluded that the overall rate of wrong site surgery was decreased approximately $62 \%$ [8]. Contrary to this report, evaluation of sentinel events after the Universal Protocol showed increased number (455) and percentage (12.8\%) of wrong-site surgery. It was not clear whether the data showed increased incidents of wrong-site surgery or an increase in the voluntary reporting system of sentinel events [22].

Other than these data, wrong exposure in spine surgery is quite common [23]. After extensive literature review regarding wrong-site surgery, Devine et al. [1] reported that the estimated rate of wrong-site surgery was from 


\section{Preoperative Check List}

Type of Anesthesia:

Patient Identification:

Surgical Site Marking:

Surgical Site Confirmation:

Time Out:

Patient identification

Surgical site confirmation

Name operation

Name of instrumentation to be used
General or Local

(1) Useful Check with Patient

(2) Bracelet

(3) Medical record or informal content

(1) done

(2) not done

(3) not applicable
(1) Left \& Right
(2) Both
(3) Single organ
(4) C Spine, T Spine, L Spine
(5) not applicable

Signature: Operator

Anesthesiologist

Circulating nurse

Fig. 3. Preoperative check list at the Severance Hospital, Yonsei University College of Medicine (Korea). 
0.09 to 4.5 per 10,000 surgeries, and among them, orthopedic procedures was the highest. Moreover, they concluded that there was no convincing data which showed decreased incidence of wrong-site surgery since the JC universal protocol [1]. Of all wrong-site surgeries, $5 \%$ to $8 \%$ involved the spine [22]. Among seventy six wrongsite surgeries reported by sixty one orthopedic surgeons, thirty one cases (43.6\%) were related to spine surgery [23]. In spine surgery, the majority of incorrect level operation was performed in the lumbar spine, followed by cervical, and thoracic spine $[23,24]$.

According to the JC data, wrong-site surgery ranked number 2 in sentinel events from 1995 to 2005 . Furthermore, analysis of the data from 2004 to 2011 showed wrong-site surgery was the most frequent sentinel event, representing 819 of 6,093 total incidents [25]. Wrong-site surgery was followed by delay in treatment (683 cases), unintended retention of a foreign body (658 cases), and operative or postoperative complications (636 cases) (Table 1).

\section{Why Does Wrong-Site Surgery Still Occur?}

In spite of SYS of AAOS, SMaX of NASS, and Universal Protocol campaign of the JC, the frequency of wrong-site surgeries is not decreasing. The causes of wrong-site surgeries include incorrect patient positioning, inadequate equipment preparation of operation room, incorrect information provided to patient and family members, failure to have informed consents, failure to make surgical markings, lack of time-out process, surgeon's failure, multiple surgeons, multiple procedures on one patient, emergency operation, etc. [1]. Many of these causes are due to poor communication, and the JC has revealed through root cause analysis that most of the wrong-site surgeries have occurred due to communication barrier and inaccurate postoperatively patient evaluation [17].

The communication failure in wrong-site surgery is categorized as the communication barrier between physician and patient and barrier among physicians and other medical personnel. The barrier between physician and patient can be somewhat resolved by providing accurate information to the patient regarding surgical site and also by having the patient participate in surgical site marking. The communication barrier among medical staff can be resolved through active expressions of not only the operator but also anesthesiologists, assistants, scrub
Table 1. Ranking the Joint Commission sentinel events (2004- 2011)

\begin{tabular}{ll}
\multicolumn{1}{c}{ Sentinel events } & $\mathrm{n}$ \\
\hline Wrong site surgery & 819 \\
\hline Delay in treatment & 683 \\
\hline Unintended retention of a foreign body & 658 \\
\hline Operative/postoperative complications & 636 \\
\hline Suicides & 600 \\
\hline Patient falls & 462 \\
\hline Other unanticipated events & 366 \\
\hline Medication error & 336 \\
\hline Criminal events & 237 \\
\hline Perinatal death/injury & 203 \\
\hline
\end{tabular}

nurses, and circulating nurses which may prevent the incidents. In order to resolve and better the communication failure among the medical staff, policies must be developed where the medical staff is educated, performance monitored, and feedback provided. Most importantly, the leadership that promotes patient safety and encourages medical staff to voluntarily participate in such campaigns must be present. The continuing frequency of wrong-site surgery may be due to the lack of leadership in hospitals.

\section{Discussion}

In 1995, when the opposite leg of a diabetic patient was amputated, the issue of wrong-site surgery became widely known to the public through the media [26]. The term, wrong-site surgery, is used in all cases where a surgery is done in wrong physical sites or in wrong patients. In spine surgery, wrong-site surgery involves the wrong level, wrong side, wrong patient surgery, and wrong procedure (the wrong procedure performed on the correct site) [1].

Instead of helping patients improve their symptoms, wrong-site surgeries cause significant legal, social, and emotional complications [24]. AAOS, NASS, and JC have developed numerous systems and led voluntary participation in order to prevent the problem. However, although the frequency is not high, wrong-site surgery still occurs in spine surgeries [23-25].

Though there are varied causative factors regarding wrong-site surgery, failure in communication is the most 
common root cause. The old resolution method of nameblame-shame, which points out an individual for blame and applies disciplinary action, does not help solve the issue, and a more systematic approach is needed. Effective communication among the medical staff is essential. For this, the leadership of the institution must develop and implement relevant policies. However, if the developed policy cannot be followed by the staff due to its complexity, it is not effective. Such policies must be simple and stream-lined, so that all staff and even patients can easily understand and implement them.

Informed consents that are too difficult for patients to understand are ineffective, and the time-out process that is too complicated for medical staff to follow is inconvenient. Therefore, when developing such policies, medical staff needs to voluntarily participate and provide input. Once the policy is developed, education must be provided so that all medical staff can be aware of the policy and implement it. The effectiveness of education must be enhanced through continuous feedback (Fig. 4). According to the policy of the Severance Hospital, Yonsei University College of Medicine anesthesiologist, ortho-
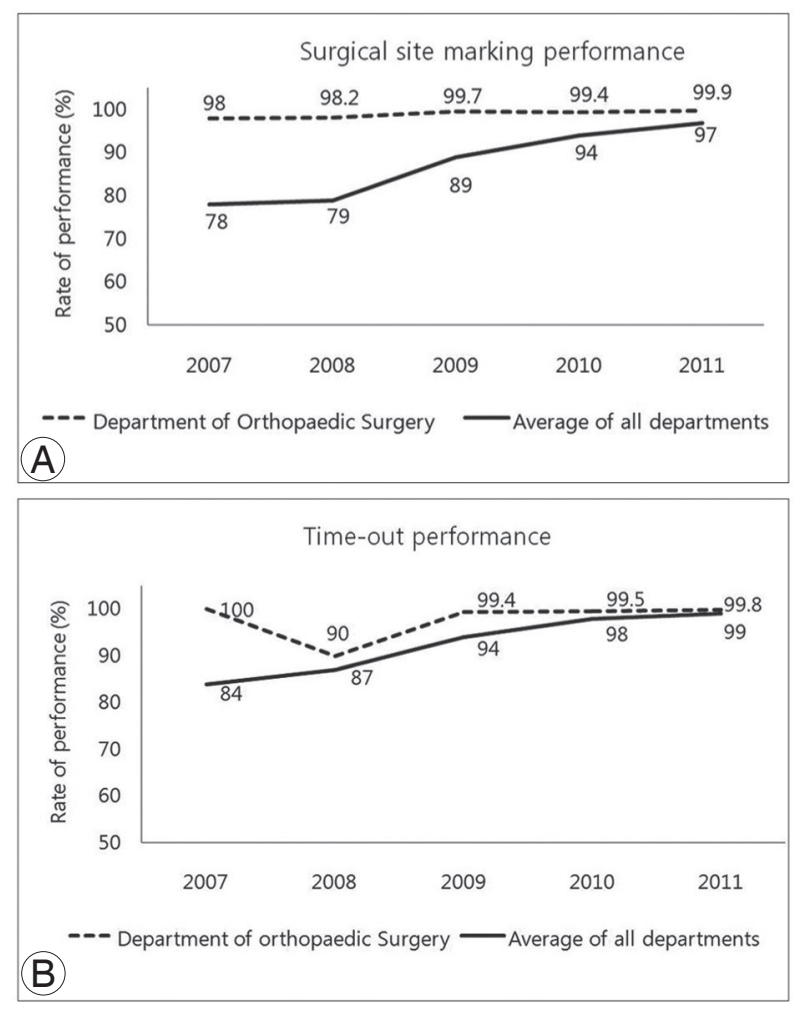

Fig. 4. Change of surgical site marking (A) and time-out (B) performance rate at the Severance Hospital. pedic resident, and the nurse participate in the process of patient verification prior to induction in operating rooms $(\mathrm{OR})$, and the time-out process is followed just prior to the incision. All team members, including the anesthesiologist, surgeon, and nurse, must participate in the timeout process, and such process of effective communication is a prerequisite in preventing wrong-site surgery.

One of the risk factors of wrong-site surgery is in the process of multiple procedures done on the same patient. In spine surgery, when anterior and posterior surgeries are done on the same patient, the time-out must be done in each surgery separately after changing the position. Emergency surgery is also a risk factor, and therefore, the time-out process must not be omitted. Since the surgeon cannot directly participate in patient's positioning and the time-out process when one surgeon is using two operation suites, it is desirable that one surgeon operates in a single operating suite if possible.

In hospitals, medical errors most frequently occur in $\mathrm{OR}$, and the most significant cause of medical errors is in communication failure. All medical staff must ensure active communication in OR. It is important to comply with all guidelines introduced above, but more importantly, efforts must be made in eliminating the hierarchy in OR. All must feel free to speak up, when a patient-safety issue is noticed. The level of hierarchy in OR is believed to be more prevalent in the Asia regions in comparison to the Western regions, and as such, OR culture where opinions are freely expressed is necessary especially in Asian countries. The traditional error resolution of name-blameshame does not pull through in preventing wrong-site surgery or any other medical errors, and efforts must be made in the team approach, emphasizing team responsibility.

\section{Conclusions}

Clearly, "To err is Human." It is definite that the problemsolving method through the system approach is essential in the prevention of wrong-site surgery. Moreover, to prevent wrong site surgery, the hospital staff must not only follow the hospital policies, but also eliminate the sense of hierarchy in OR to create a culture that promotes freedom to speak up. 


\section{Conflict of interest}

No potential conflict of interest relevant to this article was reported.

\section{Acknowledgments}

I'd like to express my sincere thanks to Grace Lim for her efforts in translating this manuscript.

\section{References}

1. Devine J, Chutkan N, Norvell DC, Dettori JR. Avoiding wrong site surgery: a systematic review. Spine (Phila Pa 1976) 2010;35:S28-36.

2. Meinberg EG, Stern PJ. Incidence of wrong-site surgery among hand surgeons. J Bone Joint Surg Am 2003;85:193-7.

3. Schweitzer KM Jr, Brimmo O, May R, Parekh SG. Incidence of wrong-site surgery among foot and ankle surgeons. Foot Ankle Spec 2011;4:10-3.

4. Wong DA, Herndon JH, Canale ST, et al. Medical errors in orthopaedics. Results of an AAOS member survey. J Bone Joint Surg Am 2009;91:547-57.

5. Antonacci MD, Eismont FJ. Neurologic complications after lumbar spine surgery. J Am Acad Orthop Surg 2001;9:137-45.

6. Eisenberg JM. Continuing education meets the learning organization: the challenge of a systems approach to patient safety. J Contin Educ Health Prof 2000;20:197-207.

7. Kohn LT, Corrigan JM, Donaldson MS. To err is human: building a safer health system. Washington DC: Institute of Medicine, National Academy Press; 1999.

8. Lewis BD. Initial evidence: reduced levels of wrong sided surgery [Internet]. Quebec, CA: Canadian Orthopaedic Association; c2009 [cited 2012 Feb 5]. Available from: http://www.coa-aco.org/library/practice-management/initial-evidence-reduced-levels-ofwrong-sided-surgery.html.

9. American Academy of Orthopaedic Surgeons. Report of the task force on wrong-site surgery. Rosemount, IL: American Academy of Orthopaedic Surgeons; 1998.

10. Wong D, Herndon J, Canale T. An AOA critical issue: medical errors in orthopaedics: practical pointers for prevention. J Bone Joint Surg Am 2002;84:2097-100.
11. American Academy of Orthopedic Surgeons. AAOS Advisory statement: wrong site surgery. Rosemont, IL: American Academy of Orthopedic Surgeons; 2003.

12. Prevention of wrong-site surgery: sign, mark, and X-ray (SMaX) [Internet]. Burr Ridege, IL: North American Spine Society; c2001 [cited 2013 Jan 17]. Available from: http://www.spine.org/Pages/PracticePolicy/ClinicalCare/SMAX/Default.aspx.

13. Wong DA. Spinal surgery and patient safety: a systems approach. J Am Acad Orthop Surg 2006;14:22632.

14. Ebraheim NA, Inzerillo C, Xu R. Are anatomic landmarks reliable in determination of fusion level in posterolateral lumbar fusion? Spine (Phila Pa 1976) 1999;24:973-4.

15. Nassr A, Lee JY, Bashir RS, et al. Does incorrect level needle localization during anterior cervical discectomy and fusion lead to accelerated disc degeneration? Spine (Phila Pa 1976) 2009;34:189-92.

16. Lindley EM, Botolin S, Burger EL, Patel VV. Unusual spine anatomy contributing to wrong level spine surgery: a case report and recommendations for decreasing the risk of preventable 'never events'. Patient Saf Surg 2011;5:33.

17. Joint Commission on Accreditation of Healthcare Organizations. Sentinel event alert. Oak Brook, IL: Joint Commission on Accreditation of Healthcare Organizations; 2001.

18. Joint Commission on Accreditation of Healthcare Organizations (JCAHO). Universal protocol for preventing wrong site, wrong procedure, wrong person surgery. Oakbrook, IL: JCAHO; 2007.

19. Joint Commission International. Joint commission international accreditation standards for hospitals [Internet]. Oak Brook, IL: Joint Commission International; c2010 [cited 2013 Jan 17]. Available from: http://www.jointcommissioninternational.org/Programs-Hospitals/.

20. World alliance for patient safety. World Health Organization (WHO) guidelines for spine surgery [Internet]. Geneva: WHO; c2008 [cited 2013 Jan 17]. Available from: http://www.gawande.com/documents/WHOGuidelinesforSafeSurgery.pdf.

21. Haynes AB, Weiser TG, Berry WR, et al. A surgical safety checklist to reduce morbidity and mortality in a global population. N Engl J Med 2009;360:491-9. 
22. Wong DA, Watters WC 3rd. To err is human: quality and safety issues in spine care. Spine (Phila $\mathrm{Pa} 1976)$ 2007;32:S2-8.

23. James MA, Seiler JG 3rd, Harrast JJ, Emery SE, Hurwitz S. The occurrence of wrong-site surgery selfreported by candidates for certification by the American Board of Orthopaedic Surgery. J Bone Joint Surg Am 2012;94(1):e2(1-12).

24. Mody MG, Nourbakhsh A, Stahl DL, Gibbs M, Al- fawareh M, Garges KJ. The prevalence of wrong level surgery among spine surgeons. Spine (Phila Pa 1976) 2008;33:194-8.

25. The Joint Commission [Internet]. Oakbrook Terrace, IL: The Joint Commission; c2013 [cited 2013 Jan 17]. Available from: http://www.jointcommission.org/.

26. Millenson ML. Pushing the profession: how the news media turned patient safety into a priority. Qual Saf Health Care 2002;11:57-63. 